\title{
Study of Multi-level Characteristics for 3D Vertical Resistive Switching Memory
}

\author{
SUBJECT AREAS: \\ ELECTRICAL AND \\ ELECTRONIC \\ ENGINEERING
}

ELECTRONIC DEVICES

Received

10 April 2014

Accepted

3 July 2014

Published

22 July 2014

Correspondence and requests for materials should be addressed to H.Q.W. (wuhq@mail. tsinghua.edu.cn)

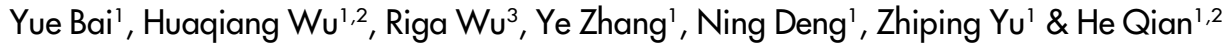

${ }^{1}$ Institute of Microelectronics, Tsinghua University, Beijing, China, 100084, ${ }^{2}$ Tsinghua National Laboratory for Information Science and Technology (TNList), Beijing, China, 100084, ${ }^{3}$ Inner Mongolia University, Hohhot, Inner Mongolia, China, 010021.

Three-dimensional (3D) integration and multi-level cell (MLC) are two attractive technologies to achieve ultra-high density for mass storage applications. In this work, a three-layer $3 \mathrm{D}$ vertical $\mathrm{AlO}_{\delta} / \mathrm{Ta}_{2} \mathrm{O}_{5-\mathrm{x}} / \mathrm{TaO}_{\mathrm{y}}$ resistive random access memories were fabricated and characterized. The vertical cells in three layers show good uniformity and high performance (e.g. $>1000 \mathrm{X}$ HRS/LRS windows, $>10^{10}$ endurance cycles, $>10^{4} \mathrm{~s}$ retention times at $125^{\circ} \mathrm{C}$ ). Meanwhile, four level MLC is demonstrated with two operation strategies, current controlled scheme (CCS) and voltage controlled scheme (VCS). The switching mechanism of 3D vertical RRAM cells is studied based on temperature-dependent transport characteristics. Furthermore, the applicability of CCS and VCS in 3D vertical RRAM array is compared using resistor network circuit simulation.

W hen the number of stored electrons reaches statistical limits, continued scaling is more and more challenging for charge-based non-volatile memory (NVM) devices ${ }^{1}$. As a solution, 3D vertical NAND technology is emerging, which achieves ultra-high bit density by stacking memory layers ${ }^{2,3}$. Recently, resistive random access memory (RRAM) has shown excellent advantages in feature size scaling and speed ${ }^{4-6}$, and is believed as one of the most promising candidates for next-generation $\mathrm{NVM}^{7-10}$. To compete with $3 \mathrm{D}$ vertical NAND, RRAM also needs to adopt 3D vertical structure. Recently several 3D RRAM studies have been reported, with many potential merits, such as simple fabrication process, low per-bit cost and good scaling capability ${ }^{11-14}$. Although recent work exhibit some improvements in endurance $\left(10^{10}\right.$ cycles $)$ or retention $\left(10^{5} \mathrm{~s}\right.$ at $\left.125^{\circ} \mathrm{C}\right)$, the studies are still preliminary and limited. For instance, the resistive switching material stack has not been widely explored which is the focus of recent optimization strategy of RRAM device performance ${ }^{15,16}$. In addition, the resistive switching window was too small $(\sim 10)$ to realize multi-level cell (MLC) applications. Moreover, the conduction and switching mechanisms for 3D vertical RRAM are still lacking of investigation.

On the other hand, the MLC operation has been widely adopted in planar NAND technology to achieve high density ${ }^{17}$. Recently, some approaches have been proposed to enable 3D vertical NAND technology ${ }^{18,19}$. However, it needs more solutions to overcome some inherent technical obstacles, such as complicated fabrication process ${ }^{20}$, charge spreading issues ${ }^{18}$ and direct coupling with neighboring cells ${ }^{21}$. In order to reach a competitive data storage density, the MLC capability is an essential requirement for 3D vertical RRAM technology. However, until now, this content has been rarely discussed.

In this work, three-layer 3D vertical $\mathrm{AlO}_{\delta} / \mathrm{Ta}_{2} \mathrm{O}_{5-\mathrm{x}} / \mathrm{TaO}_{\mathrm{y}} \mathrm{RRAM}$ cells were fabricated and characterized in detail. The devices show good resistive-switching performance and high layer-to-layer uniformity. Two operating methods are proposed to achieve 4-level MLC operations. Temperature-dependent electrical measurements are employed to investigate the resistive-switching mechanisms among four MLC levels. Furthermore, using SPICE circuit simulation method, the applicability of two MLC operating methods is evaluated and compared for 3D vertical RRAM array applications.

\section{Results and Disscussion}

Structure and fabrication process. Figure 1 (a) shows the schematic view of the three-layer $3 \mathrm{D}$ vertical $\mathrm{AlO}_{\delta} /$ $\mathrm{Ta}_{2} \mathrm{O}_{5-\mathrm{x}} / \mathrm{TaO}_{\mathrm{y}} \mathrm{RRAM}$ cell structure. The Pt pillar electrode and plane electrode serve as top electrode (TE) and bottom electrode (BE) respectively, while the resistive-switching layer is located along the sidewall between TE and $\mathrm{BE}$ vertically. The structure exhibits huge potential for future high-density integration and per-bit lithography cost reduction by increasing the stacked layers. Figure 1 (b) shows the cross-sectional TEM image of typical $3 \mathrm{D}$ vertical $\mathrm{AlO}_{\delta} / \mathrm{Ta}_{2} \mathrm{O}_{5-\mathrm{x}} / \mathrm{TaO}_{\mathrm{y}}$ RRAM devices with $3 \mathrm{um} \times 3$ um hole opening. The magnified TEM image reveal the multiple layer structure of the switching layer. The fabrication process is described in figure 1 (c) with six key processing steps: Firstly stacking $\mathrm{Si}_{3} \mathrm{~N}_{4}$ dielectric layer and Pt electrode layers alternately; then dry 

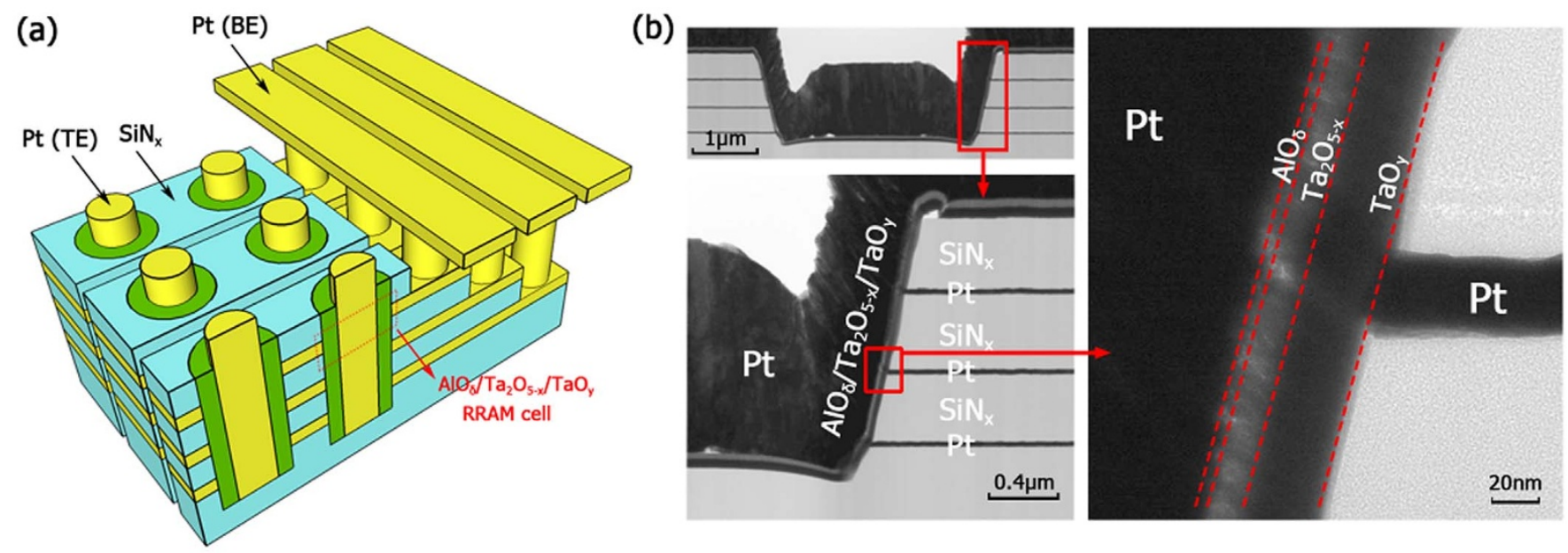

(c) (1)Deposit $\operatorname{SiN}_{x} / \mathrm{Pt}$

(2)Form staircase

(3)Etch drilled hole

(4)Deposit TMO layer

(5)Deposit TE

(6)Form slit
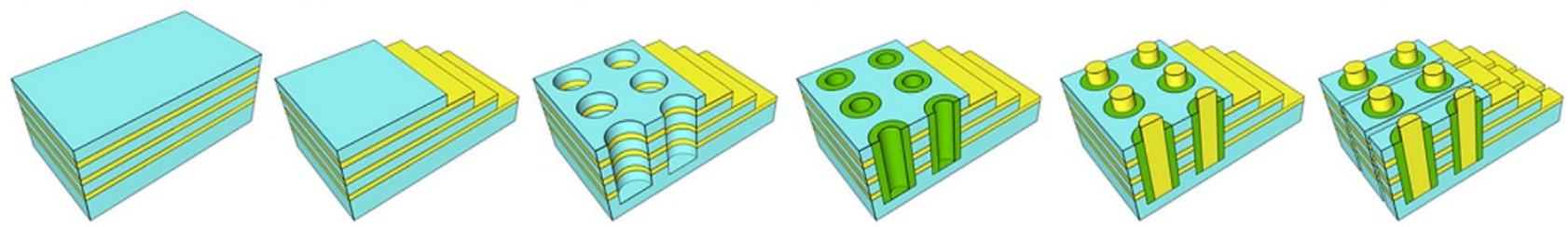

Figure $1 \mid$ (a) The schematic view of three-layer 3D vertical RRAM; (b) the cross-sectional TEM image of vertical $\mathrm{AlO}_{\delta} / \mathrm{Ta}_{2} \mathrm{O}_{5-\mathrm{x}} / \mathrm{TaO}_{\mathrm{y}} \mathrm{RRAM}$ cells; $(\mathrm{c})$ the fabrication process.

etching the plane electrode staircases; next dry etching the drilled holes and depositing the transition metal oxide (TMO) resistiveswitching layers; followed by depositing Pt as the pillar electrode; finally, slitting the plane electrodes to separate neighbouring cells. (The fabricating details are described in the method section).

Device performance. Figure 2 (a) shows typical DC I-V sweeping curves of cells in top, middle and bottom layers. Stable bipolar resistive-switching characteristics are clearly observed for devices in all three layers. These sweeping curves of vertical 3D devices are similar to previously reported planar devices ${ }^{22}$. During the SET process with negative voltage applied on TE, the device drops to a low resistive state (LRS), and with opposite voltage applied on TE for RESET process, the device switches to a high resistive state (HRS). The compliance current of $1 \mathrm{~mA}$ was enforced during SET operations. Similar to planar RRAM devices, forming operation is (a)

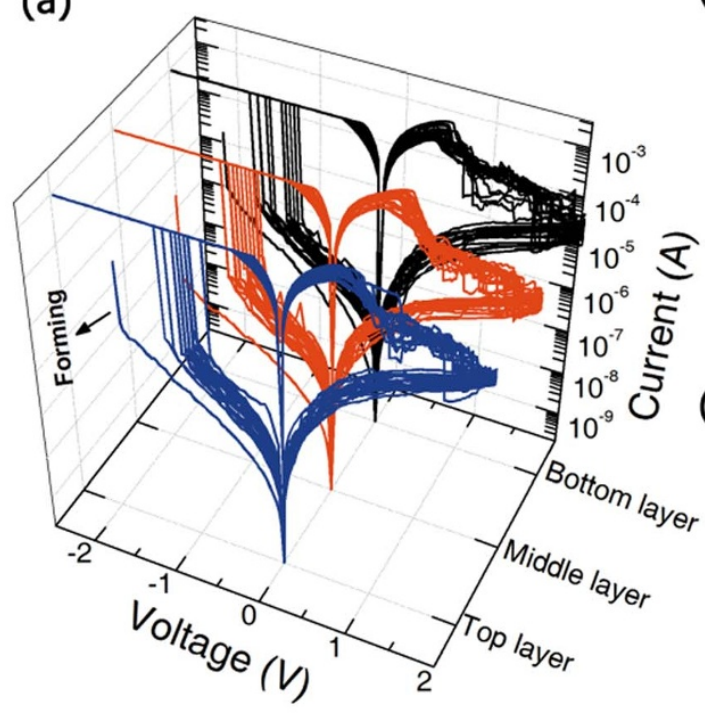

(b)

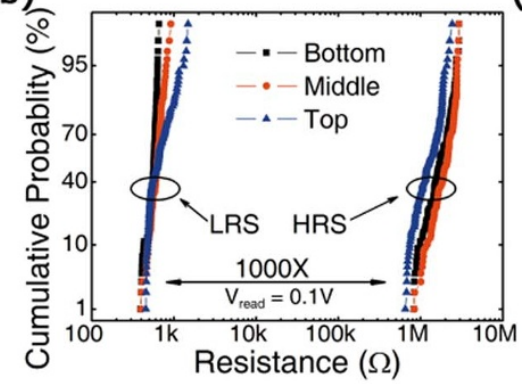

(c)

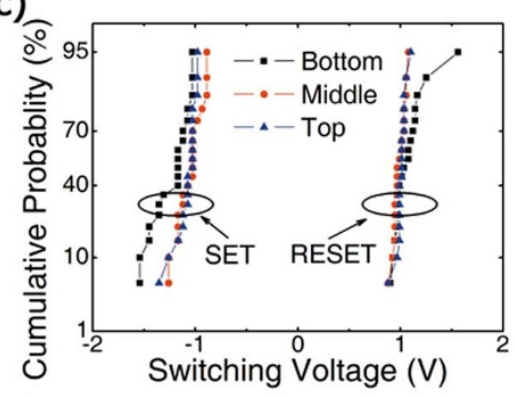

(e)

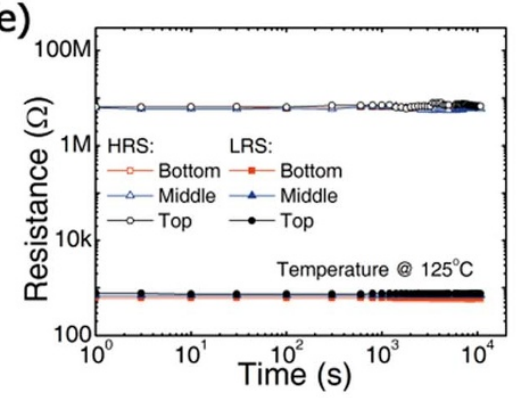

Figure $2 \mid$ The electrical performance of 3D vertical $\mathrm{AlO}_{\delta} / \mathrm{Ta}_{2} \mathrm{O}_{5-\mathrm{x}} / \mathrm{TaO}_{\mathrm{y}} \mathrm{RRAM}$ : (a) typical double DC I-V sweeping curves; (b) the HRS and LRS distributions with $1000 \times$ resistive-switching window; (c) the switching voltage distributions in SET and RESET processes; (d) $10^{10}$ switching cycles are demonstrated; (e) all cells show good retention of more than $10^{4} \mathrm{~s}$ at $125^{\circ} \mathrm{C}$. 
required to initiate the switching process as shown in Fig. 2(a). The forming voltage is about $-1.8 \mathrm{~V}$ which is slightly higher than SET voltage of $-1.2 \mathrm{~V}$.

Figure 2 (b) shows the resistance distributions of HRS and LRS. The low resistances are about $1 \mathrm{k} \Omega$ while the high resistances are about $1 \mathrm{M} \Omega$. The resistance distributions of cells in all three layers show good uniformity. Notably, the HRS/LRS resistance window is larger than 1000 times which is very suitable for MLC operations. Figure 2 (c) shows the distributions of switching voltages in SET and RESET processes. The SET voltages range from $-0.8 \mathrm{~V}$ to $-1.5 \mathrm{~V}$ while the RESET voltages are from $0.9 \mathrm{~V}$ to $2 \mathrm{~V}$.

Those 3D vertical RRAM cells also show excellent endurance and retention properties. Figure $2(\mathrm{~d})$ shows that more than $10^{10}$ switching endurance cycles were demonstrated using a pulse condition of $-1.6 \mathrm{~V}, 100 \mathrm{~ns}$ in SET operation and 1.8 V $100 \mathrm{~ns}$ for RESET operation. Figure 2 (e) shows the retention testing results. Both HRS and LRS could be kept more than $10^{4} \mathrm{~s}$ at $125^{\circ} \mathrm{C}$ without any degradation. Based on above electrical characteristics, it is concluded that the vertical RRAM cells show similar performances as planar counterpart.

The large switching window $(>1000 \mathrm{X})$ makes MLC operations possible in fabricated $3 \mathrm{D}$ vertical RRAM cells. The MLC operations could be achieved by two methods: 1) current controlled scheme (CCS) where controlling the current compliance $\mathrm{I}_{c}$ during SET processes (e.g. $5 \mu \mathrm{A}, 50 \mu \mathrm{A}, 500 \mu \mathrm{A}$ ); 2) voltage controlled scheme (VCS) where controlling the stop voltage $\mathrm{V}_{\text {stop }}$ during RESET process (e.g. 1.9 V, $2.4 \mathrm{~V}, 2.8 \mathrm{~V})$. Both of these two methods are shown in figure 3 (a). The devices in CCS share similar HRS. While, in VCS case, the devices have similar LRS. For convenience, the resistance states of 4 level MLC are defined as following: Level 1 is the LRS with resistance from $1 \mathrm{k} \Omega$ to $10 \mathrm{k} \Omega$; Level 2 and Level 3 are the intermediate states with resistance of $\sim 100 \mathrm{k} \Omega$ and $\sim 1 \mathrm{M} \Omega$, respectively; and Level 4 is the HRS with resistance of $\sim 10 \mathrm{M} \Omega$. As shown in figure 3(b), all MLC levels show good resistance distribution uniformity among three layers in the same pillar. Figure 3 (c) shows the four MLC levels DC cycling tests of three vertical RRAM sharing the same pillar electrode using VCS method. By controlling $\mathrm{V}_{\text {stop }}$, stable resistive-switching cycles among four MLC levels were demonstrated to more than 100 times without any degradation. Similar properties were observed using CCS to switch between four MLC levels.

Switching mechanism. To evaluate the MLC operation in 3D vertical $\mathrm{AlO}_{\delta} / \mathrm{Ta}_{2} \mathrm{O}_{5-\mathrm{x}} / \mathrm{TaO}_{\mathrm{y}} \mathrm{RRAM}$ structure, it is important to understand the conduction mechanism of each resistance level. Temperature dependent transport characteristics in all four MLC levels were studied, as shown in figure 4 (a)-(d). In the lowest resistance level, labeled as Level 1, the cells exhibited linear I-V relationship at all testing temperatures. The resistance increase with the temperature linearly which suggesting a metallic conduction in dominant, as shown in figure 4(a). The fitting results give a temperature coefficient of $1.7 \times 10^{-3} \mathrm{~K}^{-1}$ which is similar to previous literatures ${ }^{23,24}$. The metallic filament is formed in $\mathrm{AlO}_{\delta} / \mathrm{Ta}_{2} \mathrm{O}_{5-\mathrm{x}} / \mathrm{TaO}_{\mathrm{y}}$ vertical cells when oxygen vacancy $\left(\mathrm{V}_{\mathrm{O}}\right)$ concentration is at very high level.

For the intermediate resistance levels, i.e., Level 2 and Level 3, the electron transport is believed to be facilitated by electron hopping between a pair of $\mathrm{V}_{\mathrm{O}}$ trap sites. Nonlinear I-V relationships were observed at all testing temperatures. The conductivity $\sigma$ at low electrical field can be describe by Mott's law ${ }^{25}$ :

$$
\sigma \approx \sigma_{0} \exp \left\{-\left(\mathrm{T}_{0} / \mathrm{T}\right)^{1 / 4}\right\}
$$

The value of $\mathrm{T}_{0}$ is given by:

$$
\mathrm{T}_{0}=\frac{18 \alpha^{3}}{k_{B} N\left(E_{F}\right)}
$$

Where $\mathrm{T}$ is the absolute temperature, $\alpha$ is the range of localization wave decay lengths, $\mathrm{k}_{\mathrm{B}}$ is the Boltzmann constant, and $\mathrm{N}\left(\mathrm{E}_{\mathrm{F}}\right)$ is the density of localized states at the Fermi level. The temperature dependent electron hopping comes from the factor of $\exp \left\{-\left(\mathrm{T}_{0} /\right.\right.$ $\left.\mathrm{T})^{1 / 4}\right\}$. In figure $4(\mathrm{~b})$ and figure $4(\mathrm{c}), \ln (\mathrm{R})$ versus $\mathrm{T}^{-1 / 4}$ are plotted, where both experimental curves are well-fitted with equation (1).
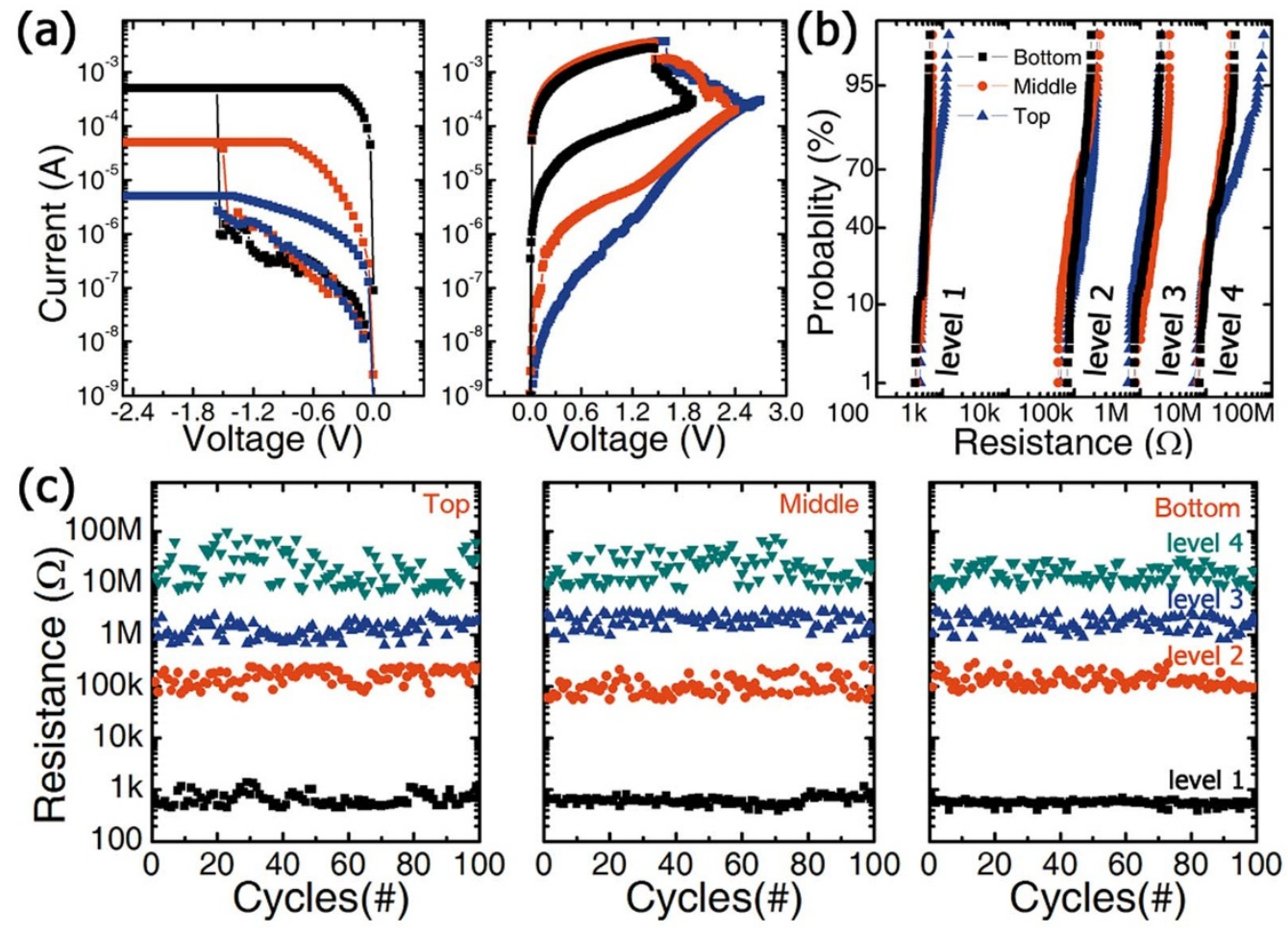

Figure 3 | (a) Two methods to achieve MLC operations: CCS and VCS; (b) resistance distributions of cells in three layers with 4 MLC levels: 1 k $\Omega$, $100 \mathrm{k} \Omega, 1 \mathrm{M} \Omega$ and $10 \mathrm{M} \Omega$; (c) stable resistive-switching DC cycles are demonstrated among 4 MLC levels. 
(a)

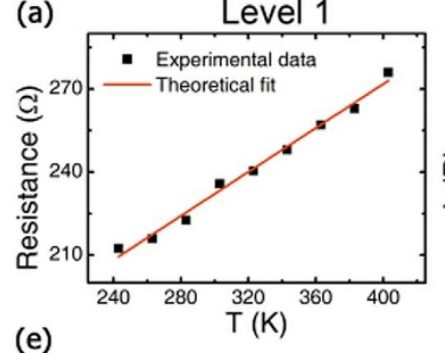

(b)
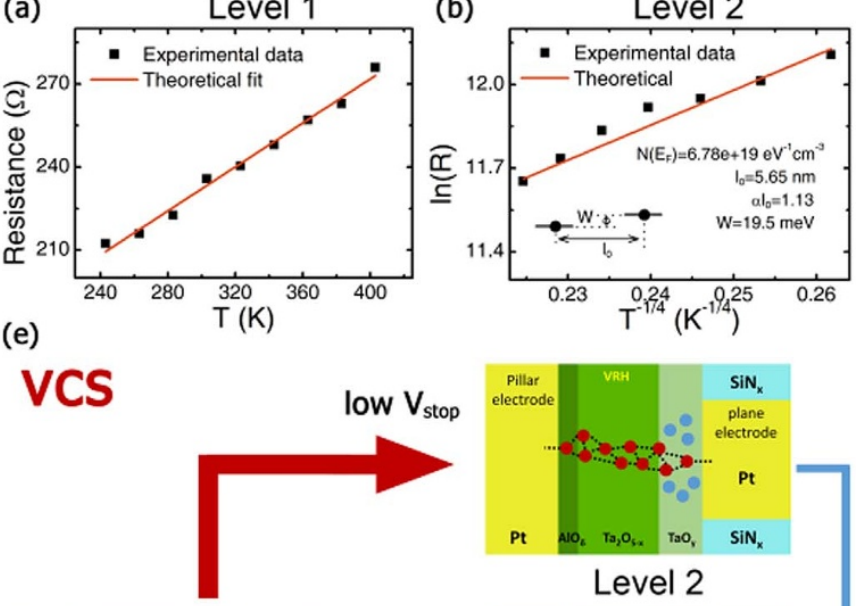

Level 2

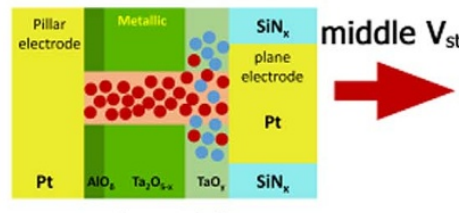

Level 1

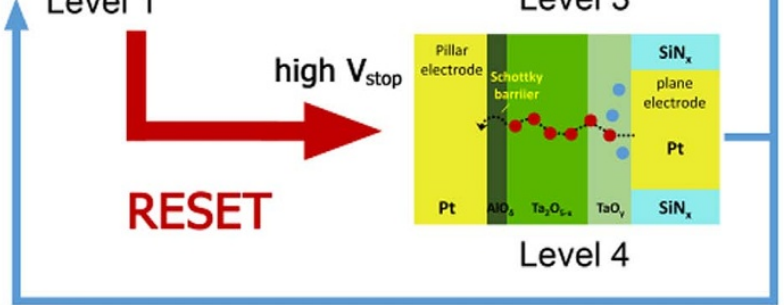

SET

same $I_{c}$

(c)

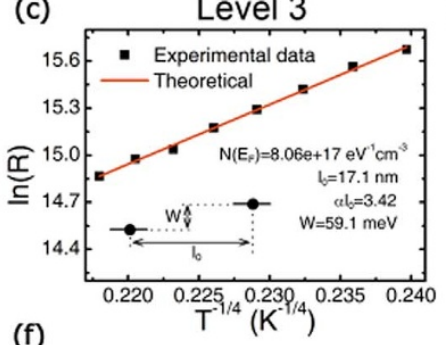

(d)

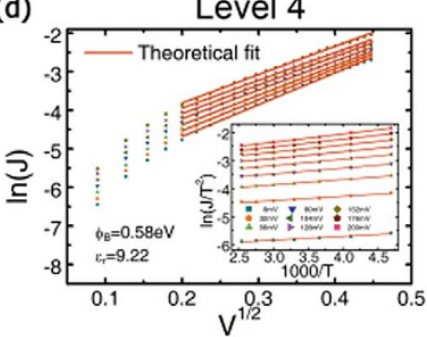

(f)
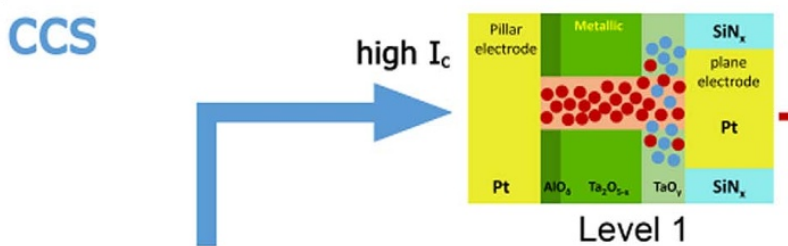

Level 1

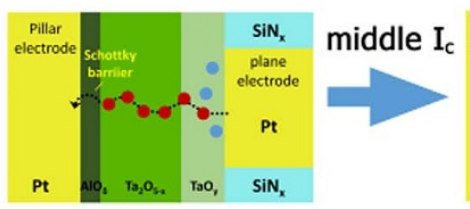

Level 4

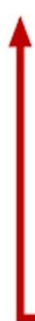

RESET

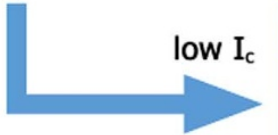

SET

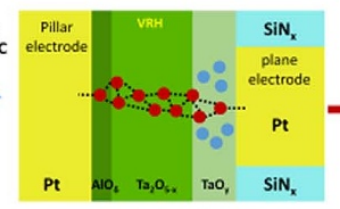

Level 2

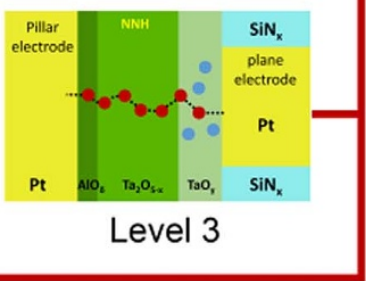

same $\mathrm{V}_{\text {stop }}$

Oxygen vacancy

\section{Oxygen ion}

Figure $4 \mid$ (a) Resistance under $0.1 \mathrm{~V}$ reading voltage vs. temperature for Level 1 ; (b-c) The $\ln (\mathrm{R}) \mathrm{vs.} \mathrm{T}^{-1 / 4}$ plot, fitted to different electron hopping models in Level 2 and Level 3. Inset shows the density of localized states at the Fermi level $\mathrm{N}\left(\mathrm{E}_{\mathrm{F}}\right)$, hopping distance $\mathrm{l}_{0}$ and activation energy $\mathrm{W}$;

(d) The $\ln (\mathrm{J})$ versus $\mathrm{V}^{1 / 2}$ plot and Schottky emission fitting in Level 4. Inset shows $\ln \left(\mathrm{J} / \mathrm{T}^{2}\right)$ versus 1/T, fitted to Schottky emission model; (e-h) The schematics of conductive mechanism: (e) the conductive filament rupture under $\mathrm{V}_{\text {stop }}$ control in VCS; (f) the conductive filament formation under $\mathrm{I}_{\boldsymbol{c}}$ control in CCS.

Therefore, $\mathrm{N}\left(\mathrm{E}_{\mathrm{F}}\right)$ could be extracted based on $\mathrm{T}_{0}$ (from the fitting slopes) and $\alpha\left(0.2 \mathrm{~nm}^{-1}\right.$ which is estimated from literatures $\left.{ }^{24-27}\right)$. Consequently, the hopping distance $\mathrm{l}_{0}$ and activation energy $\mathrm{W}$ could be obtained from:

$$
\begin{gathered}
\mathrm{l}_{0}=\left\{\frac{3}{2 \alpha(4 \pi / 3) N\left(E_{F}\right) k_{B} T}\right\}^{1 / 4} \\
W=\frac{3}{4 \pi \mathrm{l}_{0}^{3} N\left(E_{F}\right)}
\end{gathered}
$$

The fitting results in figure 4 (b) show $\mathrm{N}\left(\mathrm{E}_{\mathrm{F}}\right)=6.78 \times$ $10^{19} \mathrm{eV}^{-1} \mathrm{~cm}^{-3}, 1_{0}=5.65 \mathrm{~nm}, \mathrm{~W}=19.5 \mathrm{meV}$ in Level 2. Both $\mathrm{W}<\mathrm{k}_{\mathrm{B}} \mathrm{T}=26 \mathrm{meV}$ and $\alpha \times \mathrm{l}_{0} \approx 1.13$ are in consistent with variable-range hopping $(\mathrm{VRH})$ requirements: $\mathrm{W}<\mathrm{k}_{\mathrm{B}} \mathrm{T}$ indicates the energy levels of two remote states are close enough, and the $\alpha \times 1_{0}$ equals or less than the unity ensures the overlapping wave functions and the electrons can hop to the remote trap site within the decay length. The fitting results for the experimental curves in figure 4 (c) are different: $\mathrm{N}\left(\mathrm{E}_{\mathrm{F}}\right)=8.06 \times 10^{17} \mathrm{eV}^{-1} \mathrm{~cm}^{-3}, \mathrm{l}_{0}=17.1 \mathrm{~nm}$, and $\mathrm{W}=$ $59.1 \mathrm{meV}$. In this case, $\mathrm{W}>\mathrm{k}_{\mathrm{B}} \mathrm{T}$ and $\alpha \mathrm{l}_{0} \approx 3.42$ indicate that the localization is strong enough, and show a nearest-neighbor hopping $(\mathrm{NNH})$ characteristic is the dominant electrons transport in Level $3^{27,28}$.
For high resistance level, Level 4, much stronger temperature dependence was observed. As shown in figure 4 (d), the electrical measurement results are well-fitted with Schottky barrier emission, which is describe $\operatorname{as}^{29}$ :

$$
\mathrm{J} \approx \mathrm{A}^{*} \mathrm{~T}^{2} \exp \left\{\frac{-\mathrm{q}\left(\Phi_{B}-\sqrt{q V / 4 \pi \varepsilon_{r} \varepsilon_{0} d}\right)}{k_{B} T}\right\}
$$

Where $A^{*}$ is the effective Richardson constant, $\Phi_{B}$ is the barrier height, $\varepsilon_{\mathrm{r}}$ is the dynamic dielectric constant, $\varepsilon_{0}$ is the permittivity of vacuum space, and d is the barrier thickness. Based on device TEM image, the AlOx layer thickness is $5 \mathrm{~nm}$ which is used for $\mathrm{d}$ in equation (5). From the linear fitting of $\ln J$ vs. $\mathrm{V}^{1 / 2}$ and $\ln J / \mathrm{T}^{2}$ vs $1 / \mathrm{T}$ in figure $4(\mathrm{~d}), \Phi_{\mathrm{B}}$ and $\varepsilon_{\mathrm{r}}$ are calculated as $0.58 \mathrm{eV}$ and 9.22, similar to metal-rich $\mathrm{AlO}_{\mathrm{x}}(\sim 9)^{23,30}$. Those analysis suggest that Schottky emission become the dominant electrons conduction mechanism in Level 4.

Based on temperature dependent studies, the resistive switching mechanisms are proposed in figure 4 (e) and (f) for VCS and CCS, respectively. In VCS case, during SET operations, oxygen ions move towards to $\mathrm{TaO}_{\mathrm{y}}$ layer. The concentration of $\mathrm{V}_{\mathrm{O}}$ increases to a high level, the conductive filaments (CF) is formed and the device shows a metallic conduction property based on temperature dependent electrical tests. During RESET process, oxygen ions move back to $\mathrm{AlO}_{\delta} /$ 
$\mathrm{Ta}_{2} \mathrm{O}_{5-\mathrm{x}}$ layer under electrical field and localized joule heating could enhance the diffusion of oxygen ions from surrounding regions, and recombine with oxygen vacancies to rupture the $\mathrm{CFs}$. If $\mathrm{V}_{\text {stop }}$ is low, which is corresponding to level 2, the amount of $\mathrm{V}_{\mathrm{O}}$ is still large enough for a weak localized VRH based conduction. With the electric field further enhanced (middle $\mathrm{V}_{\text {stop }}$ ) and higher localized temperature due to joule heating, the amount of $\mathrm{V}_{\mathrm{O}}$ further decreases and the conductive mechanism changes to a strongly localized $\mathrm{NNH}$ type, as observed in Level 3. Furthermore, for a higher $\mathrm{V}_{\text {stop }}$ which is corresponding to Level 4 , when most of $V_{O}$ recombine with oxygen ions in $\mathrm{AlO}_{\delta}$ layer, a Schottky emission mode at the interface dominates the electron conduction. In contrast, the resistive switching process is different for CCS scenario, as shown in figure 4(f). Initially, the device is in Level 4 with high resistance where the limiting factor for electrons conductions is the Schottky barrier at the interface. During the SET process, the conductive filaments are formed since oxygen ions drift towards to $\mathrm{TaO}_{\mathrm{y}}$ layer under electric field. The density of $V_{O}$ could be controlled by current compliance $I_{c}$. When $I_{c}$ is increased to higher levels, cells will start switching at critical electrical field (under SET voltage), the CFs diameters will continue grow till the flowing current reach the current compliance, $\mathrm{I}_{\mathrm{c}}$. High $\mathrm{I}_{\mathrm{c}}$ will result in larger CFs in diameter which take the cell to lower resistance levels. Therefore, the conduction mechanism transfers from NNH (level 3) to VRH (level 2) and finally to metallic conduction (level 1) under different $\mathrm{I}_{\mathrm{c}}$ conditions.

The above discussion shows that the resistance is determined by the strength of CF in 3D vertical RRAM structures, similar to planar RRAM structures ${ }^{31}$. The nano-scale CF predicts an advantage in feature size scaling capability ${ }^{32}$. Furthermore, in the recent CT type 3D NAND, the direct coupling effects from neighbour cells of the same string is one critical issue. The distribution of threshold voltage $\mathrm{V}_{\text {th }}$ is deeply affected by the surrounding interference, which makes reliable MLC operation difficult to achieve ${ }^{18}$. These effects have little influence on 3D vertical RRAM structure which provides a promising future for 3D vertical RRAM structure with MLC capability.

MLC applicability analysis. For 3D vertical RRAM array, which has a different cell arrangement in comparison to planar structure, the two MLC operation methods, CCS and VCS, present different performances due to signal disturbances. In order to make an evaluation for MLC operations in 3D vertical RRAM structure, a resistor network model is constructed using SPICE method assisted by MATLAB. The basic circuit model is shown in Figure 5 (a). The 3D vertical RRAM array resembles a combination of several vertical pages. Each page is similar to a cross-bar array. Considering a $3 \mathrm{D}$ vertical RRAM with $\mathrm{M} \times \mathrm{N}$ pillars and $\mathrm{K}$ metal planes, we can describe the equivalent circuit as $\mathrm{N}$ crossbar arrays with $\mathrm{M}$ columns and $\mathrm{K}$ rows. Moreover, the interconnect resistance between neighboring cells is taken into account with a value of $1 \Omega$, following the estimation from a previous literature ${ }^{33}$. To simplify the analysis, the selector has not been taken into account yet, which will be investigated in the future works. 3D vertical RRAM array can be regarded as dividing the traditional planar array into numbers of sub-arrays. This means that, for the same amount of storage data, 3D RRAM has a smaller cross-bar array size due to stacking feature, which brings less disturbance among cells.

For SET/RESET operations, a conventional symmetric $1 / 3$ bias scheme $^{34}$ is used as shown in figure 5 (a). For the selected RRAM cell, the TE and BE are biased to V and 0 . Simultaneously, the unselected pillars and planes are biased to $1 / 3 \mathrm{~V}$ and $2 / 3 \mathrm{~V}$. In such a case, all unselected cells have the symmetric voltage drop $\pm 1 / 3 \mathrm{~V}$. It needs to point out that, during the SET operation, a current source is applied to the TE electrode of the selected cell to ensure a fixed current compliance $\mathrm{I}_{\mathrm{c}}$.

The location of the selected RRAM cell also has a notable impact on SET/RESET operations. It is found that the worst case for SET/ (a)

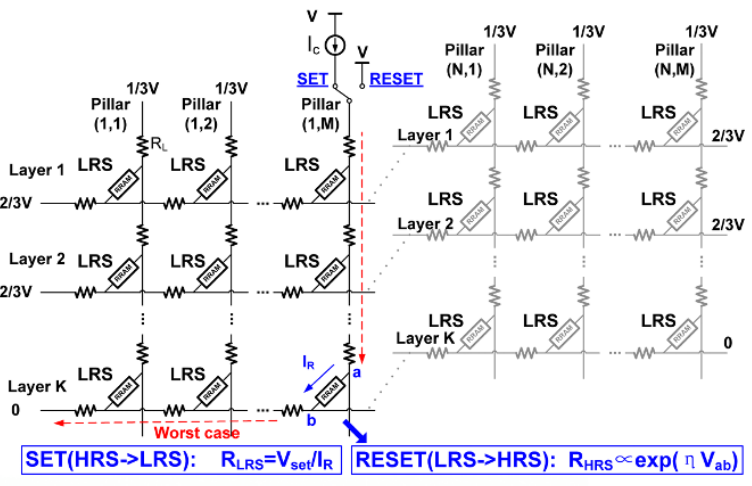

(b)

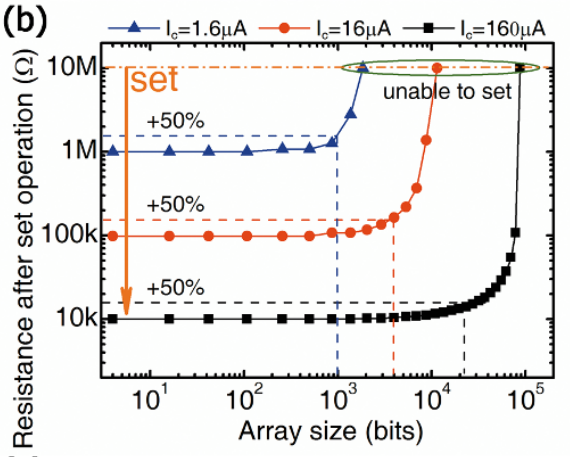

(c)

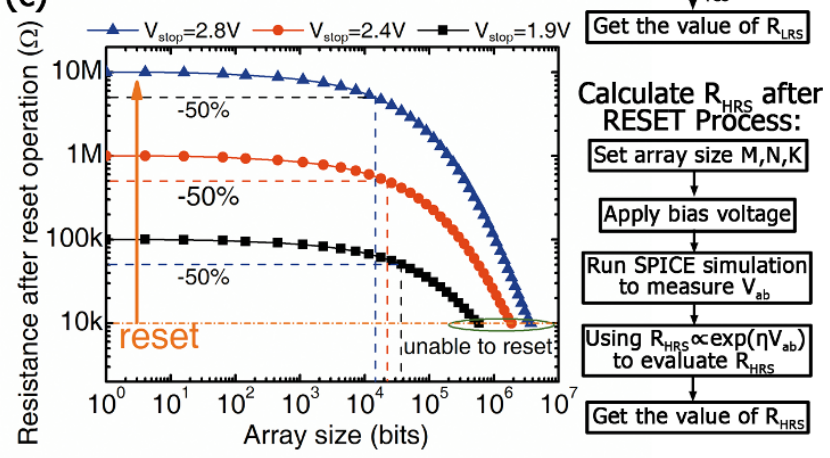

SET Process:

Set array size $M, N, K$

Set current compliance

Apply bias voltage

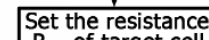

$\mathrm{R}_{\mathrm{LRS}}$ of target cell

Run SPICE simulation to measure $\mathrm{V}_{\mathrm{ab}}$

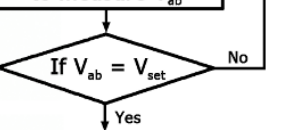

Figure $5 \mid$ (a) The equivalent circuit model of 3D vertical RRAM array with the corresponding operating method and worst case condition. (b) Three $\mathrm{R}_{\mathrm{LRS}}$ levels versus different array size, achieved by CCS method. The flowchart shows how to calculate the $\mathrm{R}_{\mathrm{LRS}}$ after SET process. (c) There $\mathrm{R}_{\text {HRS }}$ levels versus different array size, achieved by VCS method. The flowchart shows how to calculate the $\mathrm{R}_{\mathrm{HRS}}$ after RESET process.

RESET operations happens when the selected cell is at the farthest corner from the sources, as shown in the red dash line of figure 5 (a). The voltage drop and through current of this selected cell are defined as $V_{\mathrm{ab}}$ and $\mathrm{I}_{\mathrm{R}}$.

Figure 3 (a) shows that the resistive-switching is different between SET and RESET process. The current has a sharp rise at a special switching voltage $\left(\mathrm{V}_{\text {set }}\right)$ during the SET operation. Previous study shows that the $\mathrm{R}_{\mathrm{LRS}}$ is inversely proportional to the $\mathrm{I}_{\mathrm{c}}{ }^{35}$. Here, considering an ideal current compliance, the relationship could be expressed as: $R_{L R S}=V_{\text {set }} / I_{R}$. While in RESET process, the current gradually declines as the RESET voltage increases, which has also been reported in pervious work ${ }^{36}$. As a result, separate computing procedures are proposed to simulate CCS and VCS MLC operations. In CCS case, the MLC level is mainly achieved by different current compliance during SET operations, and for VCS case, the MLC level is obtained through RESET operations using different stopping voltages. The flowchart in figure 5 (b) shows the procedure to calculate three levels of $R_{L R S}$ of the selected cell using CCS method. In contrast, VCS is relatively easier to be simulated as shown in the flowchart of 
figure 5 (c). In order to get the resistance in HRS, an empirical formula is used: $R_{H R S} \propto \exp \left(\eta V_{a b}\right)$, where $\eta$ is the empirical factor extracted from our testing data, details experimental results could be referred in the supplementary information.

Figure 5 (b) shows the resistances after the SET operation in different array size. Three levels of $\mathrm{R}_{\mathrm{LRS}}$ with different current compliance are plotted. As the array size increasing, $R_{L R S}$ keeps stable at the beginning without obvious change. However a significant increase happens when array size is further enlarged. As shown in figure 5 (b), $\mathrm{R}_{\mathrm{LRS}}$ finally becomes $10 \mathrm{M} \Omega$ which is not LRS anymore. In other words, the resistance does not switch to LRS under the SET operation when array is large enough. Figure 5 (c) shows the resistance after RESET operations versus array sizes. The three levels of $\mathrm{R}_{\mathrm{HRS}}$ with different RESET stop voltages gradually decrease with the array size. Finally the $\mathrm{R}_{\mathrm{HRS}}$ stays in $10 \mathrm{k} \Omega$, with no resistive-switching under the RESET operation. An evaluation reference is defined that the maximum tolerable resistance should deviate less than $50 \%$ off the target value. Under this circumstance, VCS method could achieve much larger array size than CCS method. Only about 1000 bits array size is acceptable in CCS, while in VCS the size is more than $10^{4}$ bits. The current compliance Ic during SET process in VCS also has some effects on the array size. However the simulation results show that this effect is not the limiting factor of the array size which is shown in the supplementary information.

One possible explanation for the difference of VCS and CCS in 3D vertical RRAM array structure is proposed as following. In CCS method, the key is to control the through current of the selected cell. Under the $1 / 3$ bias scheme, the neighboring cells, which share the same pillar electrode, have $1 / 3$ voltage drop. As a results, the currents are generated through these neighboring cells. In the equivalent circuit model, parallel connected resistors are used to simulate these current. This causes the actual current on the target device is smaller than driving current to the array $\left(I_{c}\right)$, which is called the current division effect. Therefore, $\mathrm{R}_{\mathrm{LRS}}$ increase with $\mathrm{I}_{\mathrm{R}}$ decreases. With the array size getting larger and larger, this effect could be more severe and dominate the resistance of $\mathrm{R}_{\mathrm{LRS}}$. Meanwhile, during the processes to achieve three different $\mathrm{R}_{\mathrm{LRS}}$ levels, the neighboring cells have the same resistance of $10 \mathrm{k} \Omega$, which causes the division currents to remain in a common level. For a low MLC level (e.g. $10 \mathrm{k} \Omega$ ), as $\mathrm{I}_{\mathrm{c}}$ $(\sim 160 \mathrm{uA})$ is large, the current division effect is not obvious. However, for a high MLC level (e.g. $1 \mathrm{M} \Omega), \mathrm{I}_{\mathrm{c}}(\sim 1.6 \mathrm{uA})$ becomes very small. The current division effect becomes much more significant. This is why the highest MLC level (1 M $\Omega$ ) can only tolerate about 1000 bits array size. In VCS, the $\mathrm{R}_{\mathrm{HRS}}$ is only determined by the voltage drop, $\mathrm{V}_{\mathrm{ab}}$. The $\mathrm{R}_{\mathrm{HRS}}$ drop originates from voltage attenuation due to interconnect wire. When different MLC levels are targeted, the surrounding conditions are similar for the selected cells. In consequence, VCS is more suitable for 3D vertical RRAM array structure since this could support much large arrays. It is worth to point out that the simulation method for MLC applicability analysis is not unique to $3 \mathrm{D}$ vertical RRAM array. This method could be also applied to the cross point array.

\section{Conclusions}

Three-layer $3 \mathrm{D}$ vertical $\mathrm{AlO}_{\delta} / \mathrm{Ta}_{2} \mathrm{O}_{5-\mathrm{x}} / \mathrm{TaO}_{\mathrm{y}}$ RRAM cells were fabricated and characterized. The cells in top, middle and bottom layer exhibit high uniformity in SET/RESET voltages, HRS and LRS distributions. A large HRS/LRS window $(>1000 \mathrm{X})$, high endurance $\left(>10^{10}\right.$ cycles), long retention $\left(>10^{4} \mathrm{~s}\right.$ at $\left.125^{\circ} \mathrm{C}\right)$ and four level MLC operation are demonstrated. Meanwhile, a CF formation and rupture resistive-switching mechanism is proposed. This structure with MLC operation shows good potential for high density mass storage applications. Furthermore, through simulation analysis for $3 \mathrm{D}$ vertical RRAM array, it is found that VCS is more suitable than CCS method.

\section{Methods}

Fabrication process of 3D vertical $\mathrm{AlO}_{\delta} / \mathrm{Ta}_{2} \mathrm{O}_{5-\mathrm{x}} / \mathrm{TaO}_{\mathbf{y}}$ RRAM. 1) $20 \mathrm{~nm} \mathrm{Pt} \mathrm{BE}$ layer and $300 \mathrm{~nm} \mathrm{Si}_{3} \mathrm{~N}_{4}$ dielectric layer are stacked alternately by PVD and PECVD method. 2) The plane electrode staircase is formed by dry etching $\mathrm{Si}_{3} \mathrm{~N}_{4}$ layers with $\mathrm{C}_{4} \mathrm{~F}_{8} / \mathrm{CF}_{4}$ plasma and Pt layers with $\mathrm{Cl}_{2} /$ Ar plasma alternately. 3) The drilled holes to place vertical RRAM cells are dry etched with $\mathrm{Cl}_{2} / \mathrm{Ar}$ plasma. 4) $\mathrm{AlO}_{\delta} / \mathrm{Ta}_{2} \mathrm{O}_{5-\mathrm{x}} / \mathrm{TaO}_{\mathrm{y}}$ TMO resistive-switching layers are deposited on the sidewall of the drilled holes by reactive sputtering. 5) $200 \mathrm{~nm}$ Pt is deposited by PVD to form the pillar electrodes. 6) The slits of plane electrodes are dry etched to reduce the interference with neighbour cells.

Electrical measurement. The cells electrical characteristics are tested by Agilent B1500A semiconductor parameter analyzer and Agilent $81110 \mathrm{~A}$ pulse generator with Cascade Summit 11000 probe station, equipped with ERS SP72 temperature controller.

1. Goda, A. \& Parat, K. Scaling directions for 2D and 3D NAND cells. in IEEE Int. Electron Devices Meet. Tech. Dig. 2012 2.1.1-2.1.4 (2012).

2. Maeda, T. et al. Multi-stacked 1 G cell/layer Pipe-shaped BiCS flash memory. 2009 Symp. VLSI Circuits 22-23 (2009).

3. Lue, H.-T. et al. A novel dual-channel 3D NAND flash featuring both N-channel and P-channel NAND characteristics for bit-alterable Flash memory and a new opportunity in sensing the stored charge in the WL space. in IEEE Int. Electron Devices Meet. Tech. Dig. 2013 3.7.1-3.7.4 (2013).

4. Yanagida, T. et al. Scaling Effect on Unipolar and Bipolar Resistive Switching of Metal Oxides. Sci. Rep. 3 (2013).

5. Choi, B. J. et al. Electrical Performance and Scalability of Pt Dispersed $\mathrm{SiO}_{2}$ Nanometallic Resistance Switch. Nano Lett. 13, 3213-3217 (2013).

6. Hong, D. S. et al. Evolution of conduction channel and its effect on resistance switching for Au-WO3-x-Au devices. Sci. Rep. 4 (2014).

7. Kim, S., Choi, S. \& Lu, W. Comprehensive Physical Model of Dynamic Resistive Switching in an Oxide Memristor. ACS Nano 8, 2369-2376 (2014).

8. Chen, J.-Y. et al. Dynamic Evolution of Conducting Nanofilament in Resistive Switching Memories. Nano Lett. 13, 3671-3677 (2013).

9. Yang, X. \& Chen, I.-W. Dynamic-Load-Enabled Ultra-low Power Multiple-State RRAM Devices. Sci. Rep. 2 (2012).

10. Long, S. et al. Voltage and Power-Controlled Regimes in the Progressive Unipolar RESET Transition of HfO2-Based RRAM. Sci. Rep. 3 (2013).

11. Hsu, C.-W. et al. 3D Vertical $\mathrm{TaO}_{\mathrm{x}} / \mathrm{TiO}_{2}$ RRAM with over $10^{3}$ Self-Rectifying Ratio and Sub- $\mu \mathrm{A}$ Operating Current. in IEEE Int. Electron Devices Meet. Tech. Dig. 2013 10-3 (2013).

12. Yu, S., Chen, H.-Y., Gao, B., Kang, J. \& Wong, H.-S. P. HfOx-Based Vertical Resistive Switching Random Access Memory Suitable for Bit-Cost-Effective Three-Dimensional Cross-Point Architecture. ACS Nano 7, 2320-2325 (2013).

13. Hsu, C.-W., Hou, T.-H., Chen, M.-C., Wang, I.-T. \& Lo, C.-L. Bipolar RRAM With Multilevel States and Self-Rectifying Characteristics. IEEE Electron Device Lett. 34, 885-887 (2013).

14. Lee, D. et al. BEOL compatible $\left(300^{\circ} \mathrm{C}\right) \mathrm{TiN} / \mathrm{TiOx} / \mathrm{Ta} / \mathrm{TiN} 3 \mathrm{D}$ nanoscale $(\sim 10 \mathrm{~nm})$ IMT selector. in IEEE Int. Electron Devices Meet. Tech. Dig. 2013 10-7 (2013).

15. Lee, M.-J. et al. A fast, high-endurance and scalable non-volatile memory device made from asymmetric $\mathrm{Ta}_{2} \mathrm{O}_{5-\mathrm{x}} / \mathrm{TaO}_{2-\mathrm{x}}$ bilayer structures. Nat. Mater. 10, 625-630 (2011).

16. Ninomiya, T. et al. Conductive Filament Scaling of Bipolar ReRAM for Improving Data Retention Under Low Operation Current. IEEE Trans. Electron Devices 60, 1384-1389 (2013).

17. Seo, J. et al. Highly reliable M1X MLC NAND flash memory cell with novel active air-gap and $\mathrm{p}+$ poly process integration technologies. in IEEE Int. Electron Devices Meet. Tech. Dig. 2013 3.6.1-3.6.4 (2013).

18. Seo, M.-S., Lee, B.-H., Park, S.-K. \& Endoh, T. Novel Concept of the ThreeDimensional Vertical FG nand Flash Memory Using the Separated-Sidewall Control Gate. IEEE Trans. Electron Devices 59, 2078-2084 (2012).

19. Kim, W. et al. Channel-stacked NAND flash memory with layer selection by multi-level operation (LSM). in IEEE Int. Electron Devices Meet. Tech. Dig. 2013 3.8.1-3.8.4 (2013).

20. Seo, M.-S., Lee, B.-H., Park, S.-K. \& Endoh, T. A Novel 3-D Vertical FG NAND Flash Memory Cell Arrays Using the Separated Sidewall Control Gate (S-SCG) for Highly Reliable MLC Operation. 3rd IEEE Int. Mem. Workshop IMW 2011 1-4 (2011).

21. Choi, J. \& Seol, K. S. 3D approaches for non-volatile memory. 2011 Symp. VLSI Technol. VLSIT 178-179 (2011).

22. $\mathrm{Wu}, \mathrm{H}$. et al. Resistive Switching Performance Improvement of $\mathrm{Ta}_{2} \mathrm{O}_{5-\mathrm{x}} / \mathrm{TaO}_{\mathrm{y}}$ Bilayer ReRAM Devices by Inserting $\mathrm{AlO}_{\delta}$ Barrier Layer. IEEE Electron Device Lett. 35, 39-41 (2014).

23. Zhang, Y. et al. Study of conduction and switching mechanisms in $\mathrm{Al} / \mathrm{AlOx} / \mathrm{WOx} /$ $\mathrm{W}$ resistive switching memory for multilevel applications. Appl. Phys. Lett. 102, 233502-233502-4 (2013).

24. Choi, S., Yang, Y. \& Lu, W. Random telegraph noise and resistance switching analysis of oxide based resistive memory. Nanoscale 6, 400-404 (2013).

25. Mott, S. N. F. \& Davis, E. A. Electronic processes in non-crystalline materials (Clarendon Press, 1971). 
26. Khani, Z. H., Malik, M. M., Zulfequar, M. \& Husain, M. Electrical conduction mechanism in a-Se $e_{80-\mathrm{x}} \mathrm{Te}_{\mathrm{x}} \mathrm{Ga}_{20}$ films $(0<\mathrm{or}=\mathrm{x}<\mathrm{or}=20)$. J. Phys. Condens. Matter 7, 8979 (1995).

27. Goldfarb, I. et al. Electronic structure and transport measurements of amorphous transition-metal oxides: observation of Fermi glass behavior. Appl. Phys. A 107, 1-11 (2012).

28. Yang, J. J., Strukov, D. B. \& Stewart, D. R. Memristive devices for computing. Nat. Nanotechnol. 8, 13-24 (2013).

29. Emtage, P. R. \& Tantraporn, W. Schottky Emission through Thin Insulating Films. Phys. Rev. Lett. 8, 267-268 (1962).

30. Liu, Z. et al. Temperature-Dependent Charge Transport in Al/Al Nanocrystal Embedded Al2O3 Nanocomposite/p-Si Diodes. ECS Solid State Lett. 1, Q4-Q7 (2012).

31. Xu, C., Niu, D., Muralimanohar, N., Jouppi, N. P. \& Xie, Y. Understanding the Trade-offs in Multi-level Cell ReRAM Memory Design. Proc. 50th Annu. Des. Autom. Conf. 108:1-108,6 (2013).

32. Tsai, C.-L., Xiong, F., Pop, E. \& Shim, M. Resistive Random Access Memory Enabled by Carbon Nanotube Crossbar Electrodes. ACS Nano 7, 5360-5366 (2013).

33. Chen, H.-Y. et al. HfOx based vertical resistive random access memory for costeffective 3D cross-point architecture without cell selector. in IEEE Int. Electron Devices Meet. Tech. Dig. 2012 20.7.1-20.7.4 (2012).

34. Chen, Y.-C. et al. An access-transistor-free (0T/1R) non-volatile resistance random access memory (RRAM) using a novel threshold switching, selfrectifying chalcogenide device. in IEEE Int. Electron Devices Meet. Tech. Dig. 2003 37.4.1-37.4.4 (2003)

35. Ielmini, D., Nardi, F. \& Cagli, C. Universal Reset Characteristics of Unipolar and Bipolar Metal-Oxide RRAM. IEEE Trans. Electron Devices 58, 3246-3253 (2011).

36. Kim, S. et al. Physical electro-thermal model of resistive switching in bi-layered resistance-change memory. Sci. Rep. 3 (2013).

\section{Acknowledgments}

This research was supported by the National Basic Research Program (2011CBA00600), the Hi-Tech Research and Development Program (2011AA010401) and the National Natural Science Foundation $(61076115,61006067)$

\section{Author contributions}

Y.B. and H.W. planned the project and wrote the manuscript. Y.B. and R.W. fabricated the device and made electrical measurement; Y.Z. contributed to the switching mechanism. Y.B. built the circuit model and made the simulation. Y.B., H.W., N.D., Z.Y., H.Q. contributed to the conception of the experiment. All authors discussed the results and commented on the manuscript.

\section{Additional information}

Supplementary information accompanies this paper at http://www.nature.com/ scientificreports

Competing financial interests: The authors declare no competing financial interests.

How to cite this article: Bai, Y., Wu, H.Q., Wu, R., Zhang, Y., Deng, N., Yu, Z.P. \& Qian, H. Study of Multi-level Characteristics for 3D Vertical Resistive Switching Memory. Sci. Rep. 4, 5780; DOI:10.1038/srep05780 (2014).

This work is licensed under a Creative Commons Attribution-NonCommercialNoDerivs 4.0 International License. The images or other third party material in this article are included in the article's Creative Commons license, unless indicated otherwise in the credit line; if the material is not included under the Creative Commons license, users will need to obtain permission from the license holder in order to reproduce the material. To view a copy of this license, visit http:// creativecommons.org/licenses/by-nc-nd/4.0/ 